\title{
Assessment of Heterosis for Seed Yield and it's Contributing Traits in Cowpea (Vigna unguiculata L. Walp)
}

\author{
Anjali R. Talape*, P.B. Wadikar, S.H. Patil and Vaishali L. Rathod \\ College of Agriculture, Latur, Vasantrao Naik Marathwada Krishi Vidyapeeth, \\ Parbhani - 431 402(MS), India \\ *Corresponding author
}

\begin{abstract}
A B S T R A C T
The aim of the present study was to develop superior hybrids for yield and its yield components in cowpea (Vigna unguiculata L. Walp). A field experiment was undertaken to study heterosis in cowpea with objective to identifying superior hybrids for yield and yield components. The analysis was carried out with six lines and three testers by line $\mathrm{x}$ tester mating system to estimate the heterosis for yield and its attributes viz. days to 50 percent flowering, days to maturity, plant height at maturity, number of branches per plant, number of pods per plant, number of seeds per pod, pod length, 100 seed weight, harvesting index, seed yield per plant. High heterotic hybrids viz., UV-5 x Konkan Safed, UV-5 x Pusa Komal, Kashi Gauri x Konkan Safed, Kashi Nidhi x Konkan Safed and Kashi Gauri x Pusa Komal had high per se performance, heterobeltiosis and standard heterosis over check KBC-6 for yield and yield contributing traits. The highest value of heterobeltiosis and standard heterosis for UV-5 x Konkan Safed were 52.16 and 42.80 per cent respectively, for seed yield per plant.
\end{abstract}

\section{Keywords}

Cowpea, Heterosis, Line $\mathrm{x}$ tester

\section{Article Info}

Accepted:

14 November 2020

Available Online:

10 December 2020

\section{Introduction}

Cowpea (Vigna unguiculata (L) walp.) is an annual crop belongs to the family fabaceae with chromosome number $2 n=22$. It is selfpollinating crop having cleistogamous flowers, due to which variability in cowpea created through hybridization between desirable parents. The cowpea is multifunctional crop and it possesses high nutritive value. The plants are well adapted to grow under high temperature and drought and tolerate low soil fertility due to their high rated nitrogen fixation and ability to form effective symbiotic mycorrhizae, Therefore, cowpea play important role in agriculture development Nutritionally it has great potential, cowpea is a complete food minus fat its contain macronutrients and micronutrients. Among the all pulses, cowpea 
(Vigna unguiculata (L) walp.) is an important pulse crop it said "poor mans meat" locally known as chowli, southern pea, black eyed pea and labia from vedic time. There are several techniques for evaluating the varieties or cultivars or lines in terms of their combining ability and genetic architecture. Diallel, partial diallel and line $\mathrm{x}$ tester techniques are in common use. Among these, Line $\mathrm{x}$ Tester analysis technique is more suitable for large number of genotypes for understanding the genetical basis at population level (Kempthorne, 1957). It is much difficult to produce large quantity of hybrid seed in self- pollinated crop because of gene action. So there is a drastic demand to exploit heterosis in the highly self- pollinated crop for seed yield and other yield components by making all possible crosses involving the available germplasm.

\section{Materials and Methods}

The current research work was investigated at the College of Agriculture, Latur, Vasantrao Naik Marathwada Krishi Vidyapeeth, Parabhani. Nine genetically diverse cowpea genotypes comprised of six lines viz., Kashi kanchan, Kashi Gauri, GC-6, GDVC-2, Kashi Nidhi, UV-5 and 3 testers GC-3, Pusa Komal, Konkan Safed as parents and their 18 crosses (Line $\mathrm{x}$ Tester) along with one standard checks viz., KBC-6. During rabi - 2018 in line $\mathrm{x}$ tester fashion by using hand emasculation and pollination. The resulting $18 \mathrm{~F}_{1}$ hybrids along with their parents and one check were raised in Kharif - 2019 using randomized block design along with two replications. Observations were recorded on five randomly selected plants for all the characters viz., days to 50 percent flowering, days to maturity, plant height at maturity, number of branches per plant, number of pods per plant, number of seeds per pod, pod length, 100 seed weight, harvesting index, seed yield per plant.

\section{Results and Discussion}

Heterosis was calculated as per percent increase and decrease corresponding better parent and standard parents. Among 18 hybrids, three hybrids viz., GDVC-2 x Pusa Komal (-16.36\%, -12.38\%) followed by Kashi Kancha x Pusa Komal (-14.55\%, 10.48) and Kashi Gauri x Konkan Safed ($13.89 \%,-11.43 \%)$ exhibited significant and negative heterosis over better parents and standard check KBC-6 in desirable direction for character days to $50 \%$ flowering and for days to maturity the cross combination UV-5 x GC-3 (-11.52\%, -10.11\%) exhibited highest significant and negative heterosis over better parent and standard check KBC-6. It indicated that early maturity hybrids could be developed with the parental material in the present investigation. The similar results have been reported by Bhushana et al., (2000), Mehata (2000), Pal et al., (2007), Katariya et al., (2015) for days to 50\% flowering and for days to maturity Patil and Gosavi (2007) and Babariya et al., (2018). In case of plant height at maturity the crosses viz., GC-6 x Pusa Komal $(66.16 \%)$ recorded highest positively significant heterosis over the heterobeltiosis followed by Kashi Nidhi x Pusa Komal $(58.10 \%)$ and GC-6 X Konkan Safed $(32.55 \%)$. As regards to standard check, the cross UV-5 x Pusa Komal (44.53\%) had exhibited positively significant heterosis over standard check KBC-6 followed by GC-6 x Konkan Safed (37.62\%) and GC-6 x Pusa Komal $(31.91 \%)$. Cowpea is vine as well as bushy type plant due to prone against lodging therefore positive heterosis in this case is tall height is desirable (Peksen, 2004). The similar results have been reported by Sharma et al., (2010), Sarth and Reshma (2017).

The cross, GDVC-2 x Konkan Safed $(52.94 \%)$ had exhibited highest positively significant heterosis for number of branches per plant over better parent followed by Kashi 
Nidhi x Konkan Safed (26.81\%) and Kashi Gauri x GC-3 (17.53\%). Whereas, the cross GC-6 x Pusa Komal (41.33\%) had manifested positively significant heterosis over standard check KBC-6. Many earlier workers for number of branches per plant including viz., Mehata (2000), Patil and Gosavi (2007), Patel et al., (2009), Sharma et al., (2010). For number of pods per plant the crosse, UV-5 $\mathrm{x}$
Pusa Komal (39.94\%) recorded highest significant better parent heterosis followed by UV-5 x GC-3 (37.82\%) and UV-5 x Konkan safed $(24.16 \%)$ correspondingly. While, the only one cross UV-5 x Konkan Safed (26.29\%) exhibited significantly positive outcome over standard check (KBC-6). These results are in agreement with Patel et al., (2009), Sharma et al., (2010) (Table 1 and 2).

Table.1 Analysis of variance for combining ability of various quantitative characters

\begin{tabular}{|c|c|c|c|c|c|c|c|c|}
\hline Characters & $\begin{array}{l}\text { Parents } \\
\text { MSS }\end{array}$ & $\begin{array}{l}\text { Lines } \\
\text { MSS }\end{array}$ & $\begin{array}{l}\text { Tester } \\
\text { MSS }\end{array}$ & $\begin{array}{c}\text { Crosses } \\
\text { MSS }\end{array}$ & $\begin{array}{c}\text { Parents v/s } \\
\text { Crosses MSS }\end{array}$ & $\begin{array}{l}\delta^{2} \text { gca } \\
\text { (ave.) }\end{array}$ & $\delta^{2}$ sca & $\begin{array}{l}\text { Error } \\
\text { MSS }\end{array}$ \\
\hline d.f. & 8 & 5 & 2 & 17 & 1 & & & 27 \\
\hline DF & $28.097 * *$ & $23.933^{* *}$ & $43.166^{* *}$ & $39.267 * *$ & 6.259 & $5.2111 *$ & $13.4523 * *$ & 2.039 \\
\hline DM & $26.888 * *$ & $29.883^{* *}$ & $32.166^{* *}$ & $63.478 * *$ & $28.009 *$ & $7.9167 *$ & $21.9613 * *$ & 4.038 \\
\hline PH (cm) & $469.173 * *$ & $624.657 * *$ & $311.540 * *$ & $532.665 * *$ & $3950.045^{* *}$ & $37.6792 *$ & $\begin{array}{c}178.3903 * \\
*\end{array}$ & 24.659 \\
\hline NB & $3.428 * *$ & $1.896^{* *}$ & $8.211 * *$ & $3.651 * *$ & $15.075^{* *}$ & -0.3509 & $2.4529 * *$ & 0.220 \\
\hline NP & $66.167 *$ & $20.955^{* *}$ & $114.746^{* *}$ & $49.724 * *$ & $158.292 * *$ & $6.0640 * *$ & $8.5507 * *$ & 5.135 \\
\hline PL(CM) & $7.103 * *$ & $3.720 * *$ & $3.151 * *$ & $9.076^{* *}$ & $22.458 * *$ & 0.5696 & $4.3898 * *$ & 0.272 \\
\hline NS & $5.670^{* *}$ & $2.697 * *$ & $4.095 * *$ & $4.141 * *$ & 0.03169 & $0.3014^{*}$ & $1.4055^{* *}$ & 3.082 \\
\hline SW & $37.833 * *$ & $50.977 * *$ & $13.041 * *$ & $244.873 * *$ & $1312.172 * *$ & $\begin{array}{c}45.0604 * \\
*\end{array}$ & $41.5548 * *$ & 3.179 \\
\hline HI (\%) & $3.848 * *$ & $3.846^{* *}$ & $0.605 * *$ & $9.398 * *$ & $4.102 * *$ & $2.0346^{* *}$ & $1.7408 * *$ & 0.254 \\
\hline $\operatorname{SYP}(g)$ & $56.055^{* *}$ & $38.332 * *$ & 41.071 & $192.235 * *$ & $1780.228^{* *}$ & $\begin{array}{c}48.5040 * \\
*\end{array}$ & $24.6368 * *$ & 4.820 \\
\hline
\end{tabular}

$*$ and $* *$ indicated significance at 5 and $1 \%$ level respectively.

Whereas,

$\mathrm{DF}=$ Days to $50 \%$ flowering, NS =No. of seeds per pod, DM =Days to maturity, PL =Pod length, NB =No. of Branches per plant $(\%), \mathrm{PH}=\mathrm{Plant}$ height at maturity $(\mathrm{cm}), \mathrm{HI}=$ Harvesting index, $\mathrm{SW}=100$-seed weight, NP = No. of pods per plant, SYP $=$ Seed yield per plant $(\mathrm{g})$. 
Table.2 Estimates of heterosis in percentage over better parent (h1), standard check- (KBC6)(h2) for yield and yield contributing characters in cowpea

\begin{tabular}{|c|c|c|c|c|c|c|c|c|c|c|c|c|}
\hline $\begin{array}{l}\text { Sr. } \\
\text { No }\end{array}$ & Name of Crosses & $\begin{array}{c}\text { Heterosis } \\
\text { (in percent } \\
\text { over) }\end{array}$ & $\begin{array}{c}\text { Days to } 50 \% \\
\text { flowering }\end{array}$ & $\begin{array}{c}\text { Days to } \\
\text { maturity }\end{array}$ & $\begin{array}{l}\text { Plant height } \\
\text { at maturity } \\
(\mathrm{cm})\end{array}$ & $\begin{array}{c}\text { No. of } \\
\text { branches pei } \\
\text { plant }\end{array}$ & $\begin{array}{c}\text { No. of pods } \\
\text { per plant }\end{array}$ & $\begin{array}{l}\text { Pod length } \\
\text { (cm) }\end{array}$ & $\begin{array}{l}\text { Number of } \\
\text { seeds per } \\
\text { pod (gm) }\end{array}$ & $\begin{array}{c}\text { 100 Seed } \\
\text { weight }(\mathrm{gm})\end{array}$ & $\begin{array}{c}\text { Harvesting } \\
\text { index(\%) }\end{array}$ & $\begin{array}{c}\text { Seed yield } \\
\text { per plant } \\
\text { (gm) }\end{array}$ \\
\hline \multirow[t]{2}{*}{1} & \multirow{2}{*}{$\begin{array}{l}\text { KASHI KANCHAN } \\
\text { x GC-3 }\end{array}$} & $\mathrm{h}_{1}$ & 4.21 & -1.69 & $11.58^{*}$ & $-17.30 * *$ & 9.72 & $13.64 *$ & -6.07 & $-18.50 * *$ & $-34.54 * *$ & $15.63^{*}$ \\
\hline & & $\mathrm{h}_{2}$ & -5.71 & -4.26 & $12.17 *$ & -12.33 & 12.86 & $-22.55^{* *}$ & $10.04 *$ & $-50.24 * *$ & $-16.41 * *$ & -7.50 \\
\hline \multirow[t]{2}{*}{2} & \multirow{2}{*}{$\begin{array}{l}\text { KASHI KANCHAN } \\
\text { x PUSA KOMAL }\end{array}$} & $\mathrm{h}_{1}$ & $-14.55^{* *}$ & $-8.29 * *$ & $30.15^{* *}$ & 1.56 & $-14.17^{*}$ & $-22.48 * *$ & $-15.15^{* *}$ & $-24.76^{* *}$ & -0.77 & -9.83 \\
\hline & & $\mathrm{h}_{2}$ & $-10.48 * *$ & $-11.70 * *$ & $24.55^{* *} 1$ & 8.33 & -11.71 & -1.96 & $17.15^{* *}$ & $-48.27 * *$ & $32.31 * *$ & $-16.25^{* *}$ \\
\hline \multirow[t]{2}{*}{3} & \multirow{2}{*}{$\begin{array}{l}\text { KASHI KANCHAN } \\
\text { x KONKAN } \\
\text { SAFED }\end{array}$} & $\mathrm{h}_{1}$ & -2.78 & -1.60 & 3.84 & $14.47^{*}$ & 0.14 & $-25.00 * *$ & $-18.44^{* *}$ & $40.00 * *$ & $21.49 * *$ & -2.04 \\
\hline & & $\mathrm{h}_{2}$ & 0.00 & -1.60 & 7.81 & $21.33 * *$ & 3.00 & $-17.65 * *$ & -3.77 & $-16.54 * *$ & $50.77 * *$ & -3.75 \\
\hline \multirow[t]{2}{*}{4} & \multirow{2}{*}{$\begin{array}{l}\text { KASHI GAURI X } \\
\text { GC-3 }\end{array}$} & $\mathrm{h}_{1}$ & $-8.60^{*}$ & -2.91 & $14.95^{* *}$ & $17.53^{*}$ & 2.64 & $-11.96^{*}$ & -6.81 & -0.62 & $16.47 * *$ & $37.42 * *$ \\
\hline & & $\mathrm{h}_{2}$ & -3.81 & $-11.17 * *$ & 25.40 & $20.67 * *$ & 0.00 & -9.80 & 8.79 & $-38.17^{* *}$ & $48.72 * *$ & 5.12 \\
\hline \multirow[t]{2}{*}{5} & \multirow{2}{*}{$\begin{array}{l}\text { KASHI GAURI x } \\
\text { PUSA KOMAL }\end{array}$} & $\mathrm{h}_{1}$ & $-6.36^{*}$ & $6.08^{*}$ & 5.80 & $15.31^{*}$ & $-18.40 *$ & 1.55 & $-16.06^{* *}$ & $62.24 * *$ & 5.38 & $41.32 * *$ \\
\hline & & $\mathrm{h}_{2}$ & -1.90 & 2.13 & $15.41 * *$ & $23.00 * *$ & $-20.50 * *$ & $28.43^{* *}$ & $15.90^{* *}$ & $11.54 * *$ & $40.51 * *$ & $31.25^{* *}$ \\
\hline \multirow[t]{2}{*}{6} & \multirow{2}{*}{$\begin{array}{l}\text { KASHI GAURI x } \\
\text { KONKAN SAFED }\end{array}$} & $\mathrm{h}_{1}$ & $-13.89 * *$ & $-7.98 * *$ & -2.58 & 7.69 & -4.21 & 7.14 & 1.77 & $73.72 * *$ & $22.69 * *$ & $41.22 * *$ \\
\hline & & $\mathrm{h}_{2}$ & $-11.43 * *$ & $-7.98 * *$ & 6.27 & 2.67 & -2.57 & $17.65^{* *}$ & $20.08 * *$ & $8.08 *$ & $49.74 * *$ & $38.75 * *$ \\
\hline \multirow[t]{2}{*}{7} & \multirow[t]{2}{*}{ GC-6 x GC-3 } & $\mathrm{h}_{1}$ & $-7.92^{*}$ & -3.80 & -4.12 & $-13.07^{*}$ & 6.84 & $-34.45^{* *}$ & $-25.09^{* *}$ & 8.82 & $-23.69 * *$ & 5.27 \\
\hline & & $\mathrm{h}_{2}$ & $-11.43 * *$ & $-5.85^{*}$ & -3.61 & 2.00 & $-20.57 *$ & $-32.84 * *$ & $-12.55^{*}$ & $-33.56^{* *}$ & -2.56 & $-19.12^{* *}$ \\
\hline \multirow[t]{2}{*}{8} & \multirow{2}{*}{$\begin{array}{l}\text { GC- } 6 \text { x PUSA } \\
\text { KOMAL }\end{array}$} & $\mathrm{h}_{1}$ & 2.73 & 3.80 & 66.16 & $20.45^{* *}$ & $-20.25^{* *}$ & -5.43 & $-27.12 * *$ & -6.15 & $-10.77^{*}$ & $19.78^{* *}$ \\
\hline & & $\mathrm{h}_{2}$ & $7.62 *$ & 1.60 & $31.91^{* *}$ & $41.33 * *$ & -10.00 & $-19.61 * *$ & 0.63 & $-35.48^{* *}$ & $18.97 * *$ & 11.25 \\
\hline \multirow[t]{2}{*}{9} & \multirow{2}{*}{$\begin{array}{l}\text { GC-6 x KONKAN } \\
\text { SAFED }\end{array}$} & $\mathrm{h}_{1}$ & 3.70 & $5.32 *$ & $32.55^{* *}$ & $-34.66^{* * *}$ & -11.14 & $12.50^{*}$ & 6.03 & $27.10 * *$ & 7.56 & $23.41 * *$ \\
\hline & & $\mathrm{h}_{2}$ & $6.67 *$ & $5.32 *$ & $37.62 * *$ & $-23.33 * *$ & 0.29 & $23.53^{* *}$ & $2510 * *$ & $-24.23 * *$ & $31.28 * *$ & $-21.25 * *$ \\
\hline \multirow[t]{2}{*}{10} & \multirow[t]{2}{*}{ GDVC-2 x GC-3 } & $\mathrm{h}_{1}$ & $-4.26^{*}$ & 1.72 & $-22.57 * *$ & $-14.94 * *$ & -8.21 & -9.09 & $-12.90 * *$ & $20.47 * *$ & $-31.73^{* *}$ & -8.66 \\
\hline & & $\mathrm{h}_{2}$ & $-14.29 *$ & $-5.85^{*}$ & $-22.16^{* *}$ & -12.67 & $-13.71^{*}$ & -6.86 & 1.67 & $-25.87 * *$ & $-12.82^{*}$ & $-30.13^{* *}$ \\
\hline \multirow[t]{2}{*}{11} & \multirow{2}{*}{$\begin{array}{l}\text { GDVC- } 2 \text { x PUSA } \\
\text { KOMAL }\end{array}$} & $\mathrm{h}_{1}$ & $-16.36^{* *}$ & $-4.97 *$ & -11.35 & $18.12 * *$ & -1.22 & 2.71 & $-25.45 * *$ & $-15.24 * *$ & $-9.23^{*}$ & $21.00^{* *}$ \\
\hline & & $\mathrm{h}_{2}$ & $-12.38^{*}$ & $-8.51 * *$ & $-13.28^{*}$ & $26.00 * *$ & -7.14 & $29.90 * *$ & 2.93 & $-41.73 * *$ & $21.03^{* *}$ & $12.38^{*}$ \\
\hline \multirow[t]{2}{*}{12} & GDVC- $2 \mathrm{x}$ & $\mathrm{h}_{1}$ & 1.85 & 1.06 & 4.81 & $52.94 * *$ & -3.79 & 7.14 & $-11.70^{* *}$ & $42.50 * *$ & $-10.92 *$ & 10.56 \\
\hline & & $\mathrm{h}_{2}$ & 4.76 & 1.06 & 8.82 & $21.33^{* *}$ & -2.14 & $17.65^{* *}$ & 4.18 & $-12.31 * *$ & 8.72 & 8.63 \\
\hline 13 & KASHI NIDHI x & $\mathrm{h}_{1}$ & $-10.58 * *$ & -4.44 & 2.96 & 11.04 & -5.73 & $-18.18 * *$ & $-12.90 * *$ & 8.66 & $-10.84^{*}$ & -3.27 \\
\hline & & $\mathrm{h}_{2}$ & $-11.43 * *$ & $-8.51^{* *}$ & 3.51 & $14.00^{*}$ & $-15.43^{*}$ & $-16.18 * *$ & 1.67 & $-33.65 * *$ & $13.85^{*}$ & $-26.00 * *$ \\
\hline 14 & KASHI NIDHI X & $\mathrm{h}_{1}$ & -5.45 & 4.42 & $58.10^{* *}$ & $-28.13 * *$ & 4.46 & $-22.09 * *$ & $-20.91 * *$ & -2.10 & -3.08 & 2.83 \\
\hline & & $\mathrm{h}_{2}$ & -0.95 & 0.53 & $25.50 * *$ & $-23.33 * *$ & -6.29 & -1.47 & $9.21^{*}$ & $-32.69 * *$ & $29.23 * *$ & -4.50 \\
\hline 15 & KASHI NIDHI $x$ & $\mathrm{~h}_{1}$ & $-11.11 * *$ & 4.26 & -6.76 & $26.81 * *$ & 1.97 & $14.29 * *$ & 6.38 & $68.06^{* *}$ & $20.00 * *$ & $40.46^{* *}$ \\
\hline & & $\mathrm{h}_{2}$ & $14.29 * *$ & 4.26 & -3.19 & $16.67^{*}$ & 3.71 & $25.49 * *$ & $25.52 * *$ & 0.19 & $47.69^{* *}$ & $38.00 * *$ \\
\hline 16 & UV-5 x GC-3 & $\mathrm{h}_{1}$ & $-10.91^{* *}$ & $-11.52 * *$ & 5.98 & 11.69 & $37.82 * *$ & -6.70 & -0.36 & $36.23 * *$ & $-22.49 * *$ & $14.04 *$ \\
\hline & & $\mathrm{h}_{2}$ & $-6.67 *$ & $-10.11 * *$ & $20.51^{* *}$ & $14.67 *$ & $21.29 * *$ & -4.41 & $16.32 * *$ & -0.58 & -1.03 & 1.50 \\
\hline 17 & UV-5 x PUSA & $\mathrm{h}_{1}$ & -1.82 & 3.66 & $27.10^{* *}$ & $18.75 * *$ & $39.94 * *$ & $16.86^{* *}$ & -2.12 & $44.93 * *$ & $-21.54 * *$ & $56.39 * *$ \\
\hline & & $\mathrm{h}_{2}$ & 2.86 & $5.32 *$ & $44.53 * *$ & $26.67 * *$ & $23.14 * *$ & $47.79 * *$ & $35.15^{* *}$ & 5.77 & 4.62 & $45.25 * *$ \\
\hline 18 & UV-5 x KONKAN & $\mathrm{h}_{1}$ & 1.82 & 2.09 & -2.52 & 11.35 & $24.16^{* *}$ & -8.93 & 3.55 & $54.15^{* *}$ & $17.36^{* *}$ & $52.16^{* *}$ \\
\hline & & $\mathrm{h}_{2}$ & $6.67^{*}$ & 3.72 & 10.84 & 4.67 & $26.29 * *$ & 0.00 & $22.18 * *$ & $12.50 * *$ & $43.85 * *$ & $49.50 * *$ \\
\hline & $\operatorname{SE}(d)( \pm)$ & & 1.4282 & 2.0096 & 4.9658 & 0.4691 & 2.2662 & 0.5222 & 0.5209 & 1.7831 & 0.5045 & 2.1955 \\
\hline & CD@95 & & 3.0133 & 4.2399 & 10.4770 & 0.9897 & 4.7814 & 1.1018 & 1.0989 & 3.7620 & 1.0643 & 4.63322 \\
\hline & CD@99? & & 4.1394 & 5.8343 & 14.3922 & 1.3596 & 6.5682 & 1.5135 & 1.5096 & 5.1678 & 1.4621 & 6.3632 \\
\hline
\end{tabular}

$*$ and $* *$ indicated significance at 5 and $1 \%$ level respectively. 
For pod length out of 18 crosses evaluated, four and nine crosses were exhibited highest positive significant heterobeltiosis and standard heterosis over check KBC-6. The cross, UV-5 x Pusa Komal (16.86\%) and Kashi Nidhi x Konkan Safed (14.29\%), Kashi Kanchan x GC-3 (13.64) and GC-6 x Konkan Safed (12.50) recorded significant and positive heterosis over better parent and standard check KBC-6 in positive direction.

For number of seeds per pod highest significant and positive value for standard heterosis $(35.15 \%)$ was exhibited by cross UV-5 x Pusa Komal followed by Kashi Nidhi $x$ Konkan Safed (25.52\%) and GC-6 x Konkan Safed (25.10\%) respectively over standard check KBC-6. For 100 seed weight Ten crosses positively significant over better parents and three crosses positively significant over standard check. For harvesting index the crosses Kashi Kanchan $\mathrm{x}$ Konkan Safed (22.49\%) and Kashi Nidhi x Konkan Safed (20\%) exhibited highest positively significant effect for heterobeltiosis and the crosses Kashi Kanchan x Konkan Safed (50.77\%), Kashi Gauri x Konkan Safed (49.74\%) and Kashi Gauri x GC-3 (48.72\%) were expressed positive significant outcome over standard check KBC-6. High heterosis for these character over 3 type of heterosis have been reported by Chaudhari et al., (2013), Sharma et al., (2010), Patel et al., (2013) and Jyoti Kumari and Chauhan (2018) for number of seeds per pod, for 100 seed weight Bhushana et al., (2000), Patel et al., (2009) and Chaudhari et al., (2013), and for harvesting index Patel et al., (2013), Pethe et al., (2017) and Babariya et al., (2018).

For seed yield per plant the highest heterobeltiosis $(56.39 \%)$ was recorded by the cross UV-5 x Pusa Komal followed by (52.16\%) UV-5 x Konkan Safed (41.32\%) Kashi Gauri x Pusa Komal and Kashi Gauri x Konkan Safed. The value of standard heterosis for hybrids ranged from -30.13 per cent (GDVC-2 x GC-3) to 49.50 per cent (UV-5 x Konkan Safed). The highest heterosis over standard check $(49.50 \%)$ was exhibited by the cross UV-5 x Konkan Safed followed by (45.25) UV-5 x Pusa Komal, (38.75\%) Kashi Gauri x Konkan Safed. The similar findings were also in accordance with previous worker Bhushana et al., (2000), Mehata (2000), Patil and Gosavi (2007), Chaudhari et al., (2013), Katariya et al., (2015), Raut et al., (2017), Pethe et al., (2017),

In conclusion, the magnitude of heterosis was the highest for seed yield per plant. After comparing heterotic crosses and their per seperformance for various characters, it was observed that the crosses showing high heterosis and high per se performance involved the parents possessing ten high $\mathrm{x}$ high, high $\mathrm{x}$ low, low x low combining ability parents indicating importance of additive and non-additive genetic variance. So that these potential heterotic crosses may be useful for further breeding programme. The highest value of heterobeltiosis and standard heterosis were 52.16 and 42.80 per cent respectively, for cowpea seed yield per plant.

Five crosses viz., UV-5 x Konkan Safed, UV5 x Pusa Komal, Kashi Gauri x Konkan Safed, Kashi Nidhi x Konkan Safed and Kashi Gauri x Pusa Komal manifested significant and positive standard heterosis over standard check KBC-6. The above mentioned highly heterotic crosses also occupied top ranks in per se performance for cowpea seed yield. High heterotic hybrids having high per se performance as well as high and significant heterosis for other yield attributing traits, therefore, selection of these hybrids either on the basis of per se performance or on the basis of magnitude of heterotic effects for cowpea improvement would be reliable. 


\section{References}

Babariya, C. A., Dhaduk, L. K., Sapovadiya, M. H. and Ukani, J. D. (2018). Heterosis and inbreeding depression for seed yield and its components in cowpea (Vigna unguiculata L. Walp.). Int. e-J., 7(2): 156-166.

Bhushana, $\mathrm{H}$.O.., Viswanattha, K. P., Arunachalam, P. and Halesh, G. K. (2000). Heterosis in Cowpea [Vigna unguiculata (L.) Walp.] for seed yield and its attributes. Crop Res. 19(2): 277-280.

Chaudhari, S. B., Naik, M. R., Patil, S. S. and Patel, J. D. (2013a). Heterosis in cowpea for seed yield and its attributes over different environment. Trends in Biosciences., 6(4): 464-466.

Jyoti Kumari and Chauhan, D. A. (2018). Estimation of heterosis for green pod yield and attributing characters in cowpea (Vigna unguiculata L. Walp.). Int. J. Curr. Microbiol. App. Sci., 7(7): 3400-3413.

Kempthorne, O. (1957). An introduction of genetic statistics. John Willey \& Sons Inc. New York, USA: 468-473.

Katariya, H. M., Parmar, V. L., Ahir, S. D., Solanki, H. V. and Maghera, Y. V. (2015). Hybrid vigour for pod yield and seed protein content in cowpea (Vigna unguiculata L. Walp). Int. e-J., 4(4): 338-347.

Mehta, D. R. (2000). Comparison of observed and expected heterosis and inbreeding depression in four cowpea crosses. Indian Journal of Agric. Res., 34(2): 97-101.

Pal, A. K., Kumar, S. and Maurya, A. N. (2007). Genetic study for earliness in cowpea (Vigna unguiculata L. Walp.). Ind. J. of Horticulture, 64(1): 63-66.

Patel, S. J., Desai, R. T., Bhakta, R. S., Patel, D.
U., Kodappully, V. C. and Mali, S. C. (2009). Heterosis studies in cowpea (Vigna unguiculata L. Walp). Legume Res. 32(3): 199-202.

Patel, B. N, Desai, R. T., Patel, B. N. and Koladiya, P. B. (2013). Combining ability study for seed yield in cowpea (Vigna unguiculata L. Walp.). The Bioscan, 8(1):139-142.

Patil, H. E. and. Gosavi, U. S. (2007). Heterosis for yield and yield contributing characters in cowpea (Vigna unguiculata L. Walp.). Int. J. Agric. Sci., 3(2): 326-328.

Peksen Erkut and Artik Cengiz (2004).Comparison of Some Cowpea (Vigna unguiculata L. Walp ) Genotype from Turkey for Seed Yield Related Characters. Journal of Agronomy 3 (2): 137-140.

Pethe, U. B., Dodiya, N. S., Bhave S. G, Dadheech, A. and Meghawal, D. R. (2017). Heterosis for yield and yield related traits in cowpea (Vigna unguiculata L. Walp). J. of Pharmacognosy and Phytochemistry, 6(6): 1247-1249.

Raut, D. M., Tamnar, A. B., Burungale, S. V. and Badhe P. L. (2017a). Heterosis studies in cow pea (Vigna unguiculata L. Walp.) Int. J. Current. Microbial. App. Sci., 6(7): 15871593.

Sarath, P. S. and Reshma, T. (2017). Heterosis in Cowpea (Vigna unguiculata L. Walp) for selected traits. Int. J. Curr. Microbiol. App. Sci., 6(7).

Sharma, D., Mehta, N., Trivedi, J. and Gupata, C. R. (2010). Heterosis combining ability and genetic divergence in cowpea (Vigna Unguiculata L. Walp). Vegetable Sci., 37(2): 156-159.

\section{How to cite this article:}

Anjali R. Talape, P.B. Wadikar, S.H. Patil and Vaishali L. Rathod. 2020. Assessment of Heterosis for Seed Yield and It's Contributing Traits in Cowpea (Vigna unguiculata L. Walp). Int.J.Curr.Microbiol.App.Sci. 9(12): 1782-1787. doi: https://doi.org/10.20546/ijcmas.2020.912.211 